\title{
Efficiency of wave impeding barrier in pipeline construction under earthquake excitation using nonlinear finite element analysis
}

\author{
FATIH GOKTEPE ${ }^{1}$, H SERDAR KUYUK ${ }^{2}$ and ERKAN CELEBI ${ }^{1, *}$ \\ ${ }^{1}$ Department of Civil Engineering, Sakarya University, Sakarya 54187, Turkey \\ ${ }^{2}$ Berkeley Seismological Laboratory, UC Berkeley, 94720, Berkeley, USA \\ e-mail: fgoktepe@sakarya.edu.tr; skuyuk@seismo.berkeley.edu; \\ ecelebi@sakarya.edu.tr
}

MS received 28 June 2012; revised 24 July 2013; accepted 6 November 2013

\begin{abstract}
Earthquakes have caused colossal casualties and severe damages to engineering structures and especially leading to substantial economic loss to the underground structures and/or infrastructures. Pipelines are one of most important component of lifeline engineering. For instance, the Southern Caucasus- Eastern Turkey energy corridors are formed by several key pipelines carrying crude oil and natural gas from Azerbaijan, via Georgia, to world markets through Mediterranean Sea. Many project accomplished recently and construction of new corridors are still going on. They should be protected from earthquake disaster especially when they pass through high seismicity zones. The installation of wave impeding barriers (WIB) below the vulnerable infrastructures as pipelines established in soft soil can be used to reduce the effect of the earthquake induced ground borne vibrations. In this paper, a WIB as artificial bedrock based on the cut-off frequency of a soil layer over bedrock is proposed as isolation measurement in order to mitigate the dynamic response of the buried pipelines under earthquake strong ground motion. The computational simulation of the wave propagation problem is directly achieved by employing nonlinear 2D finite element modelling for prediction of screening performance of WIB on the dynamic response of vibrating coupled soil-pipeline system. Energy absorbing boundaries along the truncated interfaces of the unbounded nature of the underlying soil media are implemented in the time domain along with Newmark's integration. An extensive parametric investigation and systematic computations are performed with different controlling parameters. The obtained numerical results point out that WIB can be very promising as an isolator to protect pipelines when they establish for a certain depth.
\end{abstract}

Keywords. Wave impeding barrier; FE analysis; infrastructures; earthquake motion; non-linear soil.

*For correspondence 


\section{Introduction}

Excessive dynamic loading during strong ground motion causes in enormous stress and deformation on underground structures in active tectonic regions. Last decade, shallowly buried pipelines are utilized for a wide range of key applications, such as natural gas/oil transmission, telecommunication and water supply. Damages to small pipelines were observed in some earthquakes such as 1964 Niigata and Alaska earthquakes however fewer damages were generally reported to large underground structures compared to surface structures. On the other hand, real performance of recent project of new energy corridors such as Southern Caucasus-Eastern Turkey natural gas pipelines still unknown under strong ground motion. These vital underground structures will affect the environment extensively and the countries that depend on them. Sustainability, durability and security of these pipelines are very critical for all earth habitants. Thus, recently, earthquake-induced damages followed by severe earthquakes and earthquake corresponding resistance design have received considerable attention by the authors (Hall \& O'Rourke 1991; Liu \& Song 2005) in many countries.

There are different ways to be considered as vibration mitigation measures which should be part of the planning stage of project for an effective protection of the infrastructures from groundborne vibrations induced by incident seismic waves as: i) by modifying the wave dissipation characteristics of the soil deposit underneath of pipeline, and ii) by partially interrupting the spreading of waves into the understructure or by providing the structure more damping by means of installation certain devices such as additional base isolation systems which can be very costly. It is also possible to modify the dynamic transmitting behaviour of local sub-soil through a complex mechanism of wave reflection and mode alteration by constructing a suitable wave barrier at the bottom of pipeline (Goktepe et al 2010, 2011a, b, c; Celebi et al 2011; Celebi \& Goktepe 2012).

By the appearance of high performance computers and digital simulation tools, improvements in instrumentation and control equipment, wide range of experimental data, and consequently rapid progress in knowledge of computational structural mechanics and soil dynamics, several numerical methods with different degree of accuracy for modelling of unbounded nature of the underlying media have been performed to provide a substantial framework for soil-structure interaction (SSI) analysis under the effect of the incident seismic waves. Various numerical techniques for analysing the influences of soil heterogeneity and layering on the vibration screening by means of trench and wave impeding barriers are investigated by the authors (Beskos et al 1986; Leung et al 1991; Klein et al 1997; Takemiya 1998a, b; Chouw \& Schmid 1999; Adam \& Chouw 2001; Pflanz et al 2002; Adam \& Estorff 2005; Celebi et al 2006) to compare with the few experimental studies which are carried out full scale tests on site and laboratory model investigations only for particular cases (Woods 1968; Haupt 1981; Ahmad \& Al-Hussaini 1991; Forchap \& Verbic 1994; Celebi et al 2009).

A great number of numerical studies have been performed on the pipeline-soil interaction subject by using different models, i.e., continuous finite element model, three-dimensional FE model (Daiyan et al 2011a; Kouretzis et al 2006; Trifonov \& Cherniy 2010). To verify and/or calibrate the models used in the numerical analysis shaking table and centrifuge tests were conducted (Da Ha et al 2010; Daiyan et al 2011b). Furthermore, in the numerical analysis for wave propagation simulation different incident angles were considered in most studies (Guo 2005; Kouretzis et al 2013). In problems related to seismic soil-structure interaction analysis and wave 
propagation it is important to define the realistic material properties of the local soil condition to investigate the effects of the nonlinear behaviour of the soft soil on the overall dynamic response of the system. Additionally, it is also to have adequate and effective software package available for the FE analysis, in which the unbounded nature of the underlying soil media required to carefully model by using special artificial boundaries along the truncated interfaces. This can be performed using equivalent-linear methods to approximate the effects of soil non-linearity (FLUSH, SASSI). However, the effect of nonlinear behaviour of the surrounding soil on the seismic response of infrastructures has not been fully addressed in the literature. To further demonstrate in practical applications and to show the solutions of this type of problems to structural engineers, a comprehensive parametric analysis and systematic calculations have been conducted with various controlling parameters to evaluate the seismic response of the vibrating soil-pipeline system.

Earthquake-induced wave propagation problem with soil-structure interaction effects is directly analysed by employing a two-dimensional (2D) finite element model under plane-strain condition with elasto-plastic Mohr-Coulomb constitutive law. The proposed numerical model adapted with simulation of the real earthquake ground excitations completely considers the radiation damping condition along the fictitious interface of the unbounded media and directly predicts the reduction efficiency of the WIB proposed as passive vibration screen on the buried pipeline response.

\section{Computational model of the proposed soil-pipeline system}

The considered representative SSI model adapted with simulation of earthquake input and its computational procedure in the time domain are performed by the computer program 2D Plaxis code (Brinkgreve et al 2002) based on the Finite Element Method (FEM) to analyse the seismic behaviour of the infrastructure, and to examine isolation efficiency of WIB on pipeline to be protected. The mesh geometry and the discretization size for the numerical models as well as radiation damping are important parameters in this research. The underlying soil medium for plastic deformations and yielding is simulated by a Mohr-Coulomb soil behaviour under planestrain conditions.

\subsection{Implementation of Mohr-Coulomb soil model and absorbent boundaries in FE analysis}

The material model of considered soil based on linear elastic-perfectly plastic behaviour with a yield surface according to the Mohr-Coulomb (MC) failure criterion is implemented in its exact form in the geotechnical finite element code Plaxis. The yield surface for the MC criterion has the form of a non-uniform pyramid with hexagonal section defined by function $F(\sigma, \varphi)$, which is not affected by plastic straining. The soil is initially expected to be elastic and the model generates normal and shear stresses at all Gauss-points within the mesh. These stresses are then compared with the MC failure criterion. If the stresses at a particular Gauss-point lie within the MC failure envelope then that location is assumed to remain elastic, otherwise to be yielding.

It is seen that the MC failure criterion indicates the same trends as the conventional triaxial laboratory test data. The input plasticity parameters required for collapse mechanisms predicted by the MC model are respectively the internal friction angle $\varphi$, the soil cohesion $c$, and the dilatancy angle $\psi$, which is used to describe the flow rule as a realistic irreversible change in volume during shearing. Considering planar deformation only in elasto-plastic behaviour, with the $(\mathrm{x}, \mathrm{y})$ coordinate system, the yield function with non associated flow rule and the related 
plastic potential function in terms of the major and minor principal effective stresses can be written in the following form (Smith \& Griffith 1982):

$$
\begin{gathered}
f(\sigma, \varphi)=\left|\sigma_{i}-\sigma_{j}\right|-\left(\sigma_{i}+\sigma_{j}\right) \sin \varphi-c \cos \varphi, \\
g(\sigma, \psi)=\left|\sigma_{i}-\sigma_{j}\right|-\left(\sigma_{i}+\sigma_{j}\right) \sin \psi,
\end{gathered}
$$

where

$$
\sigma_{i}=\frac{\sigma_{i i}+\sigma_{j j}}{2}+\sqrt{\left(\frac{\sigma_{i i}+\sigma_{j j}}{2}\right)^{2}+\sigma_{i j}^{2}}, \sigma_{j}=\frac{\sigma_{i i}+\sigma_{j j}}{2}-\sqrt{\left(\frac{\sigma_{i i}+\sigma_{j j}}{2}\right)^{2}+\sigma_{i j}^{2}} .
$$

The principal relation based on the theory of small strain plasticity is that a strain rate tensor is composed of elastic and a plastic portion given as:

$$
\partial \boldsymbol{\varepsilon}_{i j}=\partial \boldsymbol{\varepsilon}_{i j}{ }^{e}+\partial \boldsymbol{\varepsilon}_{i j}^{p},
$$

in which $\boldsymbol{\varepsilon}_{i j}$ is the symmetric component of the velocity gradient tensor. In perfect plasticity, plastic strains occur when the yield criterion and the consistency equation are satisfied:

$$
\partial f=\left(\frac{\partial f}{\partial \sigma_{i j}}\right)^{T} \partial \sigma_{i j}=0
$$

where $f$ is the yield function and $\partial \sigma_{i j}$ is the infinitesimal stress increment tensor. The stress and strain vectors are respectively introduced according to the related components in planar system as:

$$
\boldsymbol{\sigma}=\left[\sigma_{x} \sigma_{y} \tau_{x y}\right]^{\mathrm{T}}, \boldsymbol{\varepsilon}=\left[\varepsilon_{x} \varepsilon_{y} 2 \varepsilon_{x y}\right]^{\mathrm{T}} .
$$

As an elastic stress increment is associated to an elastic strain increment considering the Hooke's law, use of Eq. (4) provides the following relation as:

$$
\partial \boldsymbol{\sigma}_{i j}=\boldsymbol{D}^{i j k l} \partial \boldsymbol{\varepsilon}_{i j}-\boldsymbol{D} \partial \boldsymbol{\varepsilon}_{i j}^{p}
$$

where $\boldsymbol{D}$ denotes the elastic constitutive matrix for the current stress state. In this case $\boldsymbol{D}$ can be expressed as:

$$
\boldsymbol{D}=\frac{E(1-v)}{(1+v)(1-2 v)}\left[\begin{array}{ccc}
1 & \frac{v}{1-v} & 0 \\
\frac{v}{1-v} & 1 & 0 \\
0 & 0 & \frac{1-2 v}{2(1-v)}
\end{array}\right]
$$

with $E$ the Young's modulus of elasticity and $v$ Poisson's ratio of materials. For differential plasticity models, integration of the stress rates in Eq. (7) becomes a finite stress increment as:

$$
\Delta \boldsymbol{\sigma}=\boldsymbol{D} \partial \boldsymbol{\varepsilon}-\boldsymbol{D} \partial \boldsymbol{\varepsilon}^{p}=\Delta \boldsymbol{\sigma}^{e}-\Delta \boldsymbol{\sigma}^{p},
$$

where this equation indicates the assumption that a finite stress increment is composed of an elastic part followed by a plastic part. In general, plastic strain increments are given with regard to plastic potential $g$ as:

$$
\partial \boldsymbol{\varepsilon}_{i j}^{p}=\partial \lambda \frac{\partial g}{\partial \sigma_{i j}}
$$


with $\lambda$ a positive multiplier that determines the magnitude of plastic strains. The flow rule for plastic strains given in Eq. (10) is called classical associated plasticity if $g=f$ and nonassociated otherwise. Latter flow rule are commonly used for soil materials. In principle the plastic corrector is found by integrating as:

$$
\Delta \boldsymbol{\sigma}^{p}=\int_{\lambda}^{\lambda+\Delta \lambda} \boldsymbol{D} \frac{\partial g}{\partial \sigma} \partial \lambda .
$$

In elasto-plastic analysis, the infinitesimal constitutive matrix, $\boldsymbol{D}^{e p}$, which relates the stress increments and infinitesimal strain, can be derived as:

$$
\partial \boldsymbol{\sigma}_{i j}=\boldsymbol{D}^{i j k l}\left(\partial \boldsymbol{\varepsilon}_{i j}-\partial \lambda \frac{\partial g}{\partial \sigma_{i j}}\right)
$$

By substitution of Eq. (12) into Eq. (5), $\partial \lambda$ is obtained as:

$$
\partial \lambda=\frac{\left(\frac{\partial f}{\partial \sigma_{i j}}\right)^{T} D^{i j k l} \partial \varepsilon_{k l}}{\left(\frac{\partial f}{\partial \sigma_{i j}}\right)^{T} D^{i j k l} \frac{\partial g}{\partial \sigma_{k l}}} .
$$

Finally, replacing Eq. (13) into Eq. (12), the relation between infinitesimal stresses and strains become:

$$
\partial \boldsymbol{\sigma}_{i j}=\boldsymbol{D}_{\boldsymbol{e p}}{ }^{i j k l} \partial \boldsymbol{\varepsilon}_{k l} \quad \text { where } \boldsymbol{D}_{\boldsymbol{e p}}{ }^{i j k l}=\boldsymbol{D}^{i j k l}-\frac{D^{i j k l} \frac{\partial g}{\partial \sigma_{k l}}\left(\frac{\partial f}{\partial \sigma_{i j}}\right)^{T} D^{i j k l}}{\left(\frac{\partial f}{\partial \sigma_{i j}}\right)^{T} D^{i j k l} \frac{\partial g}{\partial \sigma_{k l}}} .
$$

Incorporating the elasto-plastic modulus matrix given by Eq. (14), the following equation is valid for any elastic-perfectly plastic continuum:

$$
\sigma^{i}=\sigma^{i-1}+\int_{\varepsilon^{i-1}}^{\varepsilon^{i-1}+\Delta \varepsilon^{i_{e p}}} D_{e p}{ }^{i} \partial \varepsilon_{e p}{ }^{i} .
$$

To obtain meaningful results from SSI analysis, energy absorbing conditions, which can reduce the whole unbounded soil medium to a finite region, should correctly describe the interaction forces between near and far fields in the governing equations. The dynamic equilibrium for soilstructure interaction system under plane-strain condition can be written by the following matrix differential equation:

$$
\boldsymbol{M} \ddot{u}(t)+\boldsymbol{C} \dot{u}(t)+\boldsymbol{C}^{\infty \dot{u}(t)}+\boldsymbol{K} \boldsymbol{u}(t)=\tilde{F}(t),
$$

where the mass matrix $\boldsymbol{M}$, material damping matrix $\boldsymbol{C}$ constructed based on Rayleigh damping which is formulated as a function of the mass and stiffness matrices (Zienkiewicz \& Taylor 1991; 
Hughes 1987) radiation damping matrix $\boldsymbol{C}^{\infty}$, stiffness matrix $\boldsymbol{K}$, and the vector of equivalent nodal forces $\tilde{F}(t)$ are respectively given by:

$$
\begin{aligned}
& \boldsymbol{M}=\iint_{\Omega} N^{T} N \rho \partial \Omega, \\
& \boldsymbol{C}=\alpha_{R} \boldsymbol{M}+\beta_{R} \boldsymbol{K}=\alpha_{R} \iint_{\Omega} N^{T} N \rho \partial \Omega+\beta_{R} \iint_{\Omega} B^{T} D B \partial \Omega, \\
& \boldsymbol{C}^{\infty}=\iint_{\Omega} N^{T}\left[\begin{array}{cc}
c_{1} \rho V_{p} & 0 \\
0 & c_{2} V_{s}
\end{array}\right] N \partial \Omega \\
& \boldsymbol{K}=\left(\iint_{\Omega} B^{T} D B \partial \Omega\right) \\
& \tilde{F}(t)=\boldsymbol{F}(t)+\boldsymbol{F}^{\infty}(t)=\iint_{\Omega} N^{T} b \partial \Omega+\iint_{\Gamma} N^{T} t \partial \Gamma \\
& -\left(\iint_{\Omega} N^{T}\left[\begin{array}{cc}
c_{1} \rho V_{p} & 0 \\
0 & c_{2} \rho V_{s}
\end{array}\right] N \partial \Omega\right)\left(\dot{u}_{b}-\dot{u}_{b f}\right),
\end{aligned}
$$

in which $\rho$ is the mass density of the soil, $V_{p}$ and $V_{s}$ are the pressure wave velocity and the shear wave velocity, respectively. Here, $c_{1}$ and $c_{2}$ are relaxations coefficients that have been introduced to improve the performance of the absorption at the local viscous boundaries. The multiplication of gradient operator $\boldsymbol{L}$ and interpolations functions $\boldsymbol{N}$ called as displacement field gives the strain displacement transformation matrix $\boldsymbol{B} . \boldsymbol{b}$ is the density vector of the specified body forces and $\boldsymbol{t}$ is a vector of external tractions that may be imposed to surface $\Gamma$, defined in Eq. (21). To considerably simplify the SSI analysis, special boundary conditions, which can absorb the energy waves, are specified along the truncated interfaces of the model boundaries to avoid spurious reflection of waves back into the soil medium. Hereby, $\boldsymbol{F}(t)^{\infty}$ is the force vector related to the viscous damper on the artificial boundary. $\dot{u}_{b}$ and $\dot{u}_{b f}$ are respectively the nodal velocities on the boundary and in the corresponding free field. When plasticity presents, it is more appropriate to use the incremental form of Eq. (16) and then the matrix $\boldsymbol{K}$ given by Eq. (20) should be the tangential matrix, which indicates the relation between increments of nodal displacement and the resulting increments of nodal load, as given below:

$$
K_{t}=\left(\iint_{\Omega} B^{T} D^{e p} B \partial \Omega\right),
$$

where $\boldsymbol{D}^{e p}$ is given by Eq. (14) if $f=0$ and $\partial f=0$, but is replaced by elastic constitutive matrix $\boldsymbol{D}$ if $f<0$ and $\partial f<0$. 


\subsection{Meshing and geometry}

The FE representation of the soil-infrastructure coupled system with a wave impeding block to be proposed for the vibration problem is schematically shown in figure 1 with numerical detail. To obtain the desired accuracy with a reasonable computing time and memory requirements of the dynamic response of the buried pipeline in the soft soil where the plastic deformations are expected, the finer FE mesh $\left(H_{1}=10 \mathrm{~m}, B_{1}=50 \mathrm{~m}\right)$ is used in the modelling to be capable of transmitting all the vibratory wave patterns (figure 1). This is achieved by smaller element size ( $\Delta h \leq 1.2 \mathrm{~m}$ ), which is defined by the condition that of those not exceed one eighth to one fifth of the shortest Rayleigh wavelength at the highest frequency of the significant components of the Fourier response spectrum. The time step integration has been chosen as $\Delta t=0.033$ taking into account the Courant condition for the FEM simulations (Courant et al 1967). The mesh of the remaining subzones (hereby, $H_{2}=40 \mathrm{~m}, B_{2}=160 \mathrm{~m} ; \Delta h=2.8 \mathrm{~m}$ ) has been designed to be relatively coarse than above mentioned localized domain increasing in size gradually reaching
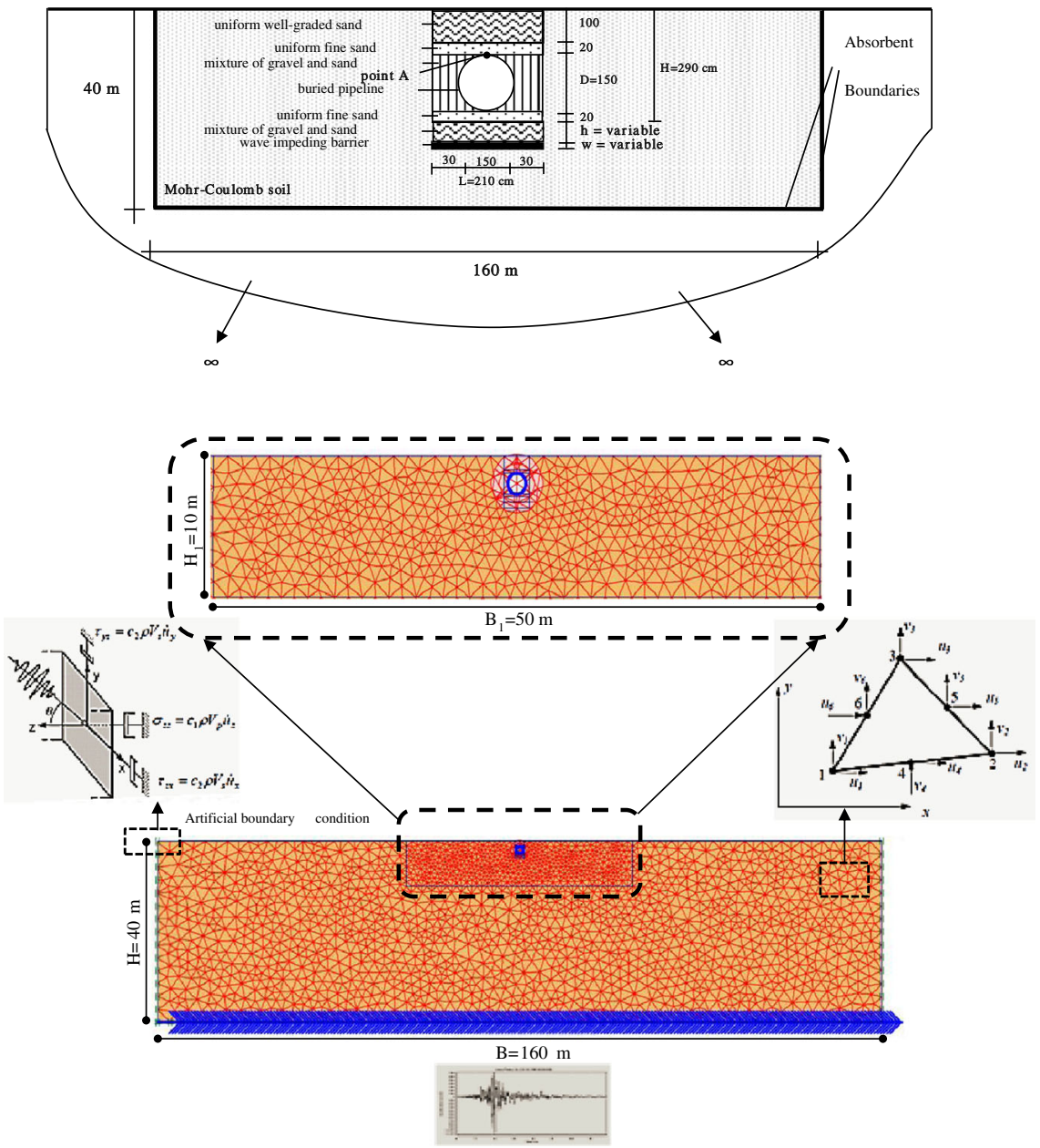

Figure 1. Schematic diagram of the considered soil-structure model by wave impeding barrier. 

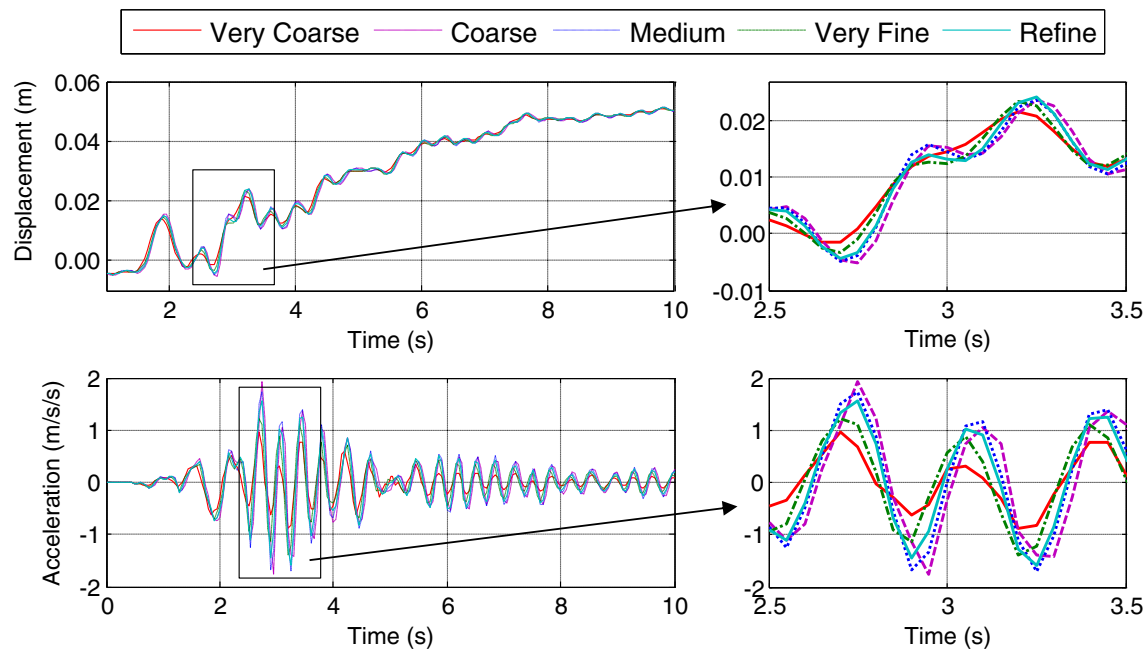

Figure 2. Displacement and acceleration responses of the model with various meshing sizes.

the value of $2.8 \mathrm{~m}$ (the maximum allowable element size in this case) for distant elements near the lower edge.

Two-dimensional 6-node triangular elements are used in the FE mesh generation of the soil region. This type of element consists of two translational degrees of freedom at each node under
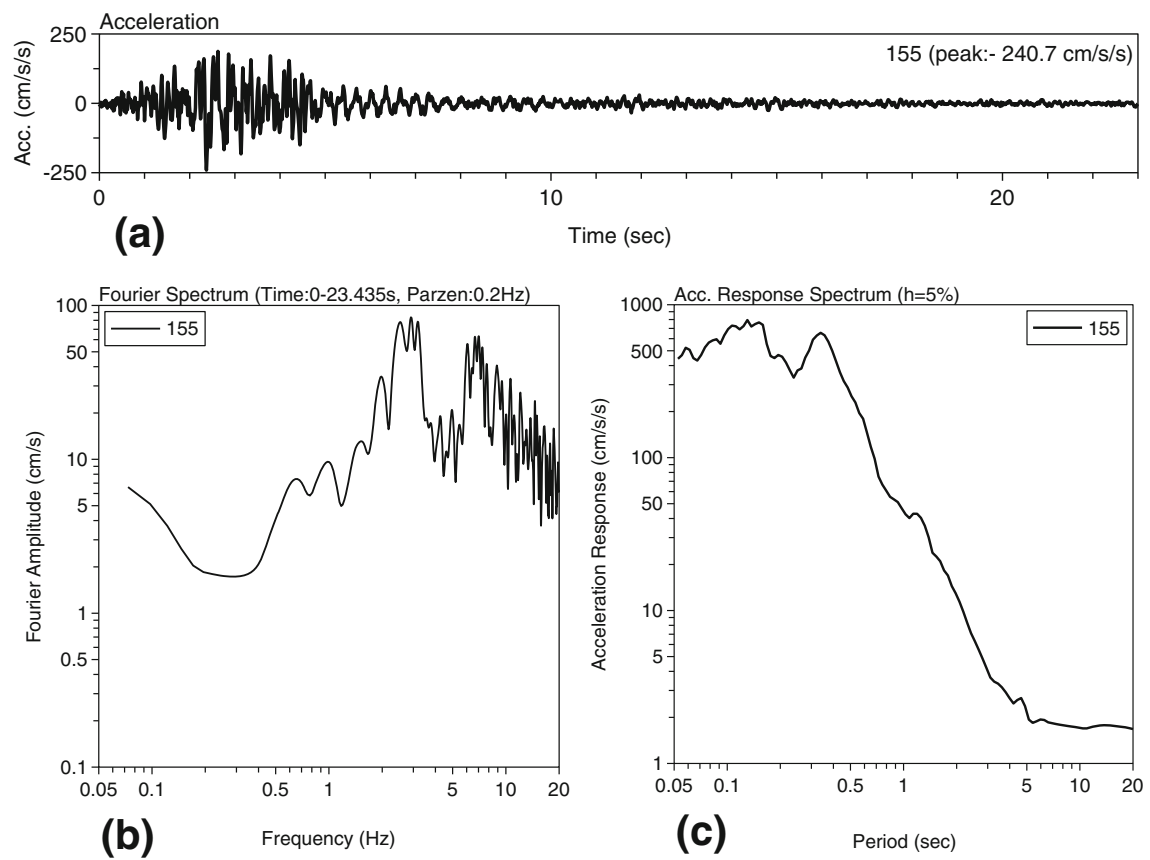

Figure 3. (a) Acceleration record of 1990 upland earthquake with a maximum 240.7 gal. (b) Fourier spectrum. (c) Acceleration response spectrum. 
Table 1. Properties of soil for undrained FE Mohr-Coulomb model (Gamber 2004).

\begin{tabular}{lccc}
\hline Parameter & Symbol & Unit & Magnitude \\
\hline Total unit weight & $\gamma$ & $\left(\mathrm{kN} / \mathrm{m}^{3}\right)$ & 16.67 \\
Mass density & $\rho$ & $\left(\mathrm{Mg} / \mathrm{m}^{3}\right)$ & 1.70 \\
Young's modulus & $\mathrm{E}$ & $(\mathrm{kPa})$ & 34500.00 \\
Shear modulus & $\mathrm{G}$ & $(\mathrm{kPa})$ & 13270.00 \\
Poisson's ratio & $\nu$ & - & 0.30 \\
Constrained modulus & $\mathrm{M}$ & $(\mathrm{kPa})$ & 46440.00 \\
Compression wave velocity & $\mathrm{Vp}$ & $\mathrm{m} / \mathrm{s}$ & 165.200 \\
Shear wave velocity & $\mathrm{Vs}$ & $\mathrm{m} / \mathrm{s}$ & 88.300 \\
Damping Rayleigh alpha factor & $\alpha$ & - & 0.001 \\
Damping Rayleigh beta factor & $\beta$ & - & 0.01 \\
Void ratio & $\mathrm{e}$ & - & 0.50 \\
Cohesion & $\mathrm{c}$ & $(\mathrm{kPa})$ & 0.00 \\
Friction angle & $\varnothing$ & $\left(^{\circ}\right)$ & 33.00 \\
Dilatancy angle & $\psi$ & $\left(^{\circ}\right)$ & 3.00 \\
Interface strength reduction factor & Rinter & - & 0.67 \\
\hline
\end{tabular}

plain strain condition. It provides a fourth order interpolation for deformed shape associated to the wave lengths in this numerical algorithm. The 5 node-plate elements with two translational and one rotational degree of freedom per nodes, representing the infrastructure, are considered to be linear elastic. The FE mesh size is selected as the lateral extent of $B=160 \mathrm{~m}$ and the total depth of $H=40 \mathrm{~m}$ as shown in figure 1 based on the results of displacement and acceleration responses of the model with various meshing sizes (figure 2). The measurement point in the model at chosen node for displacement time history response is on the top of the buried pipeline which is described as $A$.

Unless otherwise specified, the embedding depth of the pipeline is taken as $H=2.9 \mathrm{~m}$ and its diameter is assumed to be $D=1.5 \mathrm{~m}$. Only the depth $(h)$ and the thickness $(w)$ of the WIB are described to be variable parameters in the analysis of the infrastructural response. In this case the length of the WIB is considered as $L=2.1 \mathrm{~m}$ for achieving an ideal vibration reduction under economically practical.

The 1990 Upland Earthquake acceleration record was considered as input motion which can be seen acceleration (without any filtering and baseline corrections), Fourier spectrum (smoothed with $0.2 \mathrm{~Hz}$ Parzen window) and response acceleration spectrum of record showed in figure 3.

Table 2. Properties of filling soil of buried pipeline for undrained FE Mohr-Coulomb model (Zaneta 2006).

\begin{tabular}{|c|c|c|c|c|c|c|c|}
\hline Soil type & $\begin{array}{l}\text { Mass density } \\
\left(\mathrm{Mg} / \mathrm{m}^{3}\right)\end{array}$ & $\begin{array}{l}\text { Young's } \\
\text { modulus } \\
(\mathrm{kPa})\end{array}$ & $\begin{array}{c}\text { Poisson's } \\
\text { ratio (-) }\end{array}$ & $\begin{array}{l}\text { Friction } \\
\text { angle }\left(^{\circ}\right)\end{array}$ & $\begin{array}{c}\text { Cohesion } \\
(\mathrm{kPa})\end{array}$ & $\begin{array}{l}\text { Damping } \\
\text { Rayleigh } \\
\text { alpha } \\
\text { factor (-) }\end{array}$ & $\begin{array}{l}\text { Damping } \\
\text { Rayleigh } \\
\text { beta } \\
\text { factor(-) }\end{array}$ \\
\hline Mixture of gravel and sand & 2.00 & 15000 & 025 & 38.00 & 3.00 & 0.01 & 0.01 \\
\hline Uniform fine sand & 1.60 & 15000 & 0.25 & 32.00 & 0.00 & 0.01 & 0.01 \\
\hline Uniform well-graded sand & 1.80 & 20000 & 0.25 & 33.00 & 0.00 & 0.01 & 0.01 \\
\hline
\end{tabular}


Table 3. Properties of steel pipeline for FE elastic plate-element.

\begin{tabular}{lccc}
\hline Parameter & Symbol & Unit & Magnitude \\
\hline Total unit weight & $\gamma$ & $\left(\mathrm{kN} / \mathrm{m}^{3}\right)$ & 77.00 \\
Mass density & $\rho$ & $\left(\mathrm{Mg} / \mathrm{m}^{3}\right)$ & 7.85 \\
Young's modulus & $\mathrm{E}$ & $(\mathrm{kPa})$ & $2 \times 10^{8}$ \\
Damping factors & $\alpha, \beta$ & - & 0.05 \\
Poisson's ratio & $\nu$ & - & 0.30 \\
\hline
\end{tabular}

Note that, maximum acceleration of the record is 240.7 gal while dominant frequency is about $3 \mathrm{~Hz}$. The acceleration was recorded in Live Oak Reservoir-Abutment with a $5 \mathrm{~km}$ epicentral distance. This is a near field strong ground motion record with high frequency content.

Wave-impeding barrier installed in various thicknesses and depths are proposed as reduction measure to reveal the optimal geometrical properties. The essential material parameters considered in FE model for the underlying and filling soil together with steel pipeline are summarized in tables 1, 2 and 3, respectively. As a boundary condition, the pipe is considered as a free surface on the periphery of pipe and no normal stresses are imposed due to liquid flowing in the pipe.

\section{Parametric studies and numerical results}

The theoretical concept of wave isolation measures depending on cut-off frequency of the soil stratum is based on reflection, scattering and diffraction of vibration energy. In this study, a parametric investigation has been executed with respect to some key parameters to introduce the shielding performance of a wave impeding block as a passive isolation when constructed below pipeline for reducing adverse effects of earthquake vibrations.

\subsection{Effect of the WIB thicknesses, w}

First of all, effectiveness of the WIB thickness on the infrastructural vibrations is evaluated. For this case, various thicknesses changing from 10 to $100 \mathrm{~cm}$ are examined. A dimensionless parameter which is obtained from the ratio of thickness to width of trench is derived and proposed for the optimum thickness. The width of trench which is equal to $210 \mathrm{~cm}$ is assumed as summation of the radius of pipeline and both side of $30 \mathrm{~cm}$ around for placing and practicing the compaction of soil. The screening efficiency of the WIB will be evaluated in terms of $w / L$ ratios ranging from 0.05 to 0.48 for different selected values of thickness. The material characteristics

Table 4. Properties of concrete wave impeding block (WIB) for FE elastic model.

\begin{tabular}{lccc}
\hline Parameter & Symbol & Unit & Magnitude \\
\hline Total unit weight & $\gamma$ & $\left(\mathrm{kN} / \mathrm{m}^{3}\right)$ & 22.00 \\
Mass density & $\rho$ & $\left(\mathrm{Mg} / \mathrm{m}^{3}\right)$ & 2.24 \\
Young's modulus & $\mathrm{E}$ & $(\mathrm{kPa})$ & $3.7 \times 10^{7}$ \\
Damping factors & $\alpha, \beta$ & - & 0.01 \\
Poisson's ratio & $v$ & - & 0.25 \\
\hline
\end{tabular}



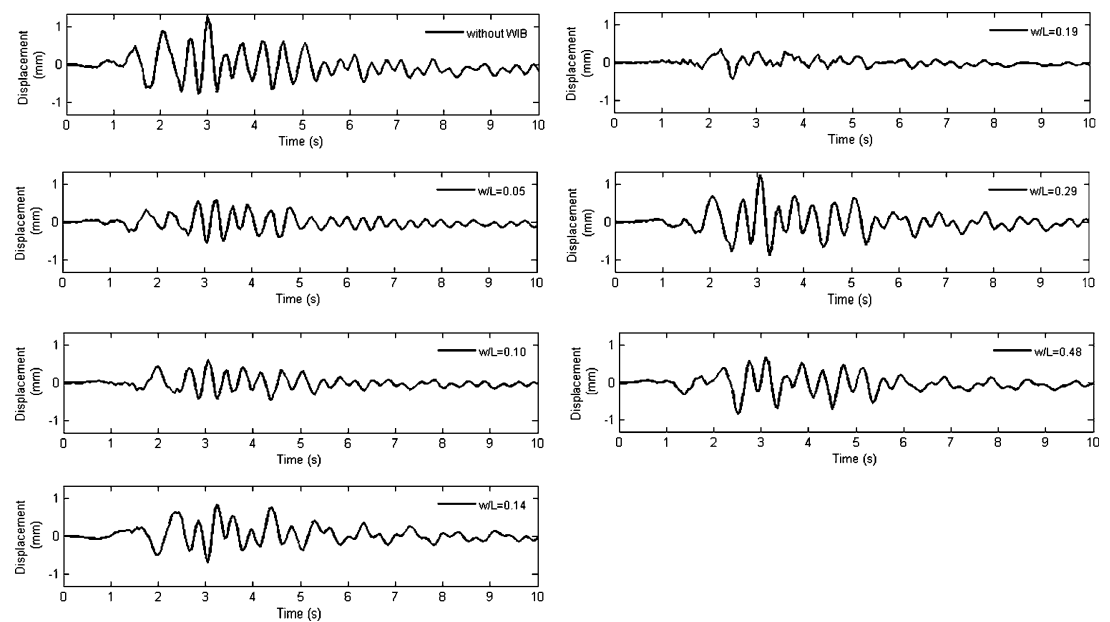

Figure 4. The vertical displacement time histories depending on $w / L$ ratios at measurement point $\mathrm{A}$.

regarded in FE model for the concrete block are given in table 4. We investigated only one type of concrete which elasticity modules $(\mathrm{E})$ is $3.7 \times 10^{7} \mathrm{kPa}$. As a parametric study, we have not looked at the effectiveness of different materials due to scope of this study, however we are further planning to examine various material properties as a WIB.

The vertical displacements time history recorded at measurement point A after dynamic analysis in case of no screen and WIB for various $w / L$ ratios are shown in figure 4 . The ratio with 0.19 results in the best attenuation considering peak ground displacement (PGD). Any thickness seems effective except for the ratio of 0.29 . The waveforms are not precisely similar; overall

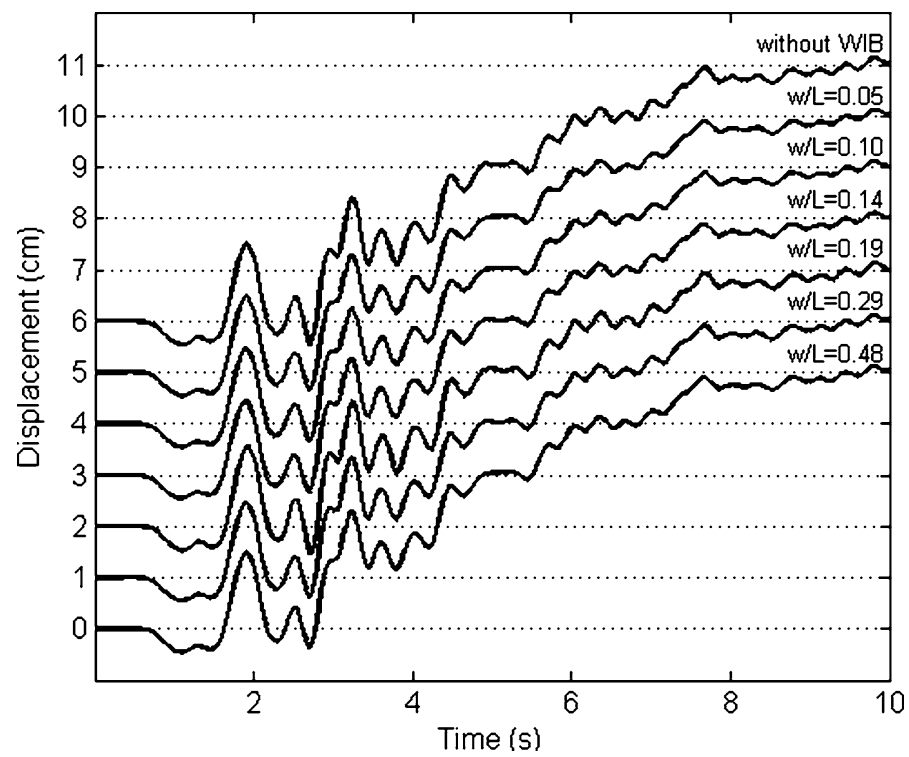

Figure 5. The horizontal displacement time histories depending on $w / L$ ratios at measurement point $\mathrm{A}$ (shifted record for $1 \mathrm{~cm}$ ). 

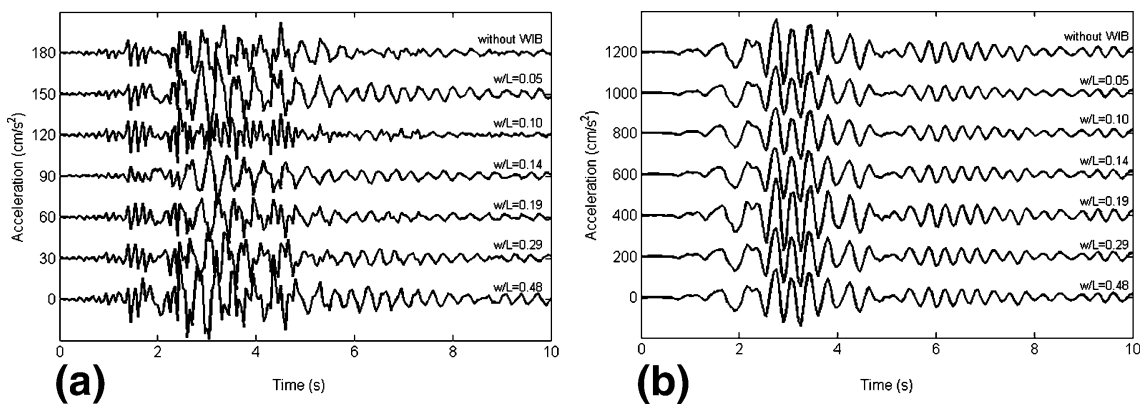

Figure 6. The resulting time histories of the (a) vertical and (b) horizontal accelerations depending on various $\mathrm{w} / \mathrm{L}$ (shifted records for $30 \mathrm{~cm} / \mathrm{s}^{2}$ and $200 \mathrm{~cm} / \mathrm{s}^{2}$ ).

envelope of waveform appeared to diminish however the peak value is almost same as without usage of WIB. However, there is almost no difference in horizontal displacements, as displayed in figure 5. Displacements are plotted one centimeter shifted for better visualization. About $5 \mathrm{~cm}$ dislocation is seen in each case which means, the WIB has no effect in horizontal displacement. As expected, the WIB embedded horizontally at a suitable depth inside the soil, is only effective in mitigation of the earthquake excitation generated structural vibrations with predominant frequencies below the cut-off frequency of the soil stratum which no oscillation eigen modes can be induced. Because of the restricted length of the WIB, the spreading of waves into the surrounding area cannot be completely obstructed, which is commonly stated as the leaking problem.

The resulting time histories of the vertical and horizontal accelerations are plotted in figure 6 . Top waveforms indicate acceleration without WIB where the waveforms underneath demonstrates with different thickness of WIB. Best result is achieved for 0.10 of $w / L$ ratio in terms of peak ground acceleration (PGA) together with the higher frequency content among others. Long period motion seems to be filtered out most at $20 \mathrm{~cm}$ thickness. Similar tendency is seen for small thickness less than 0.19 of $w / L$ ratio. PGA in horizontal direction is much larger than the PGA of vertical acceleration as expected. High frequency content is also less than the vertical records.
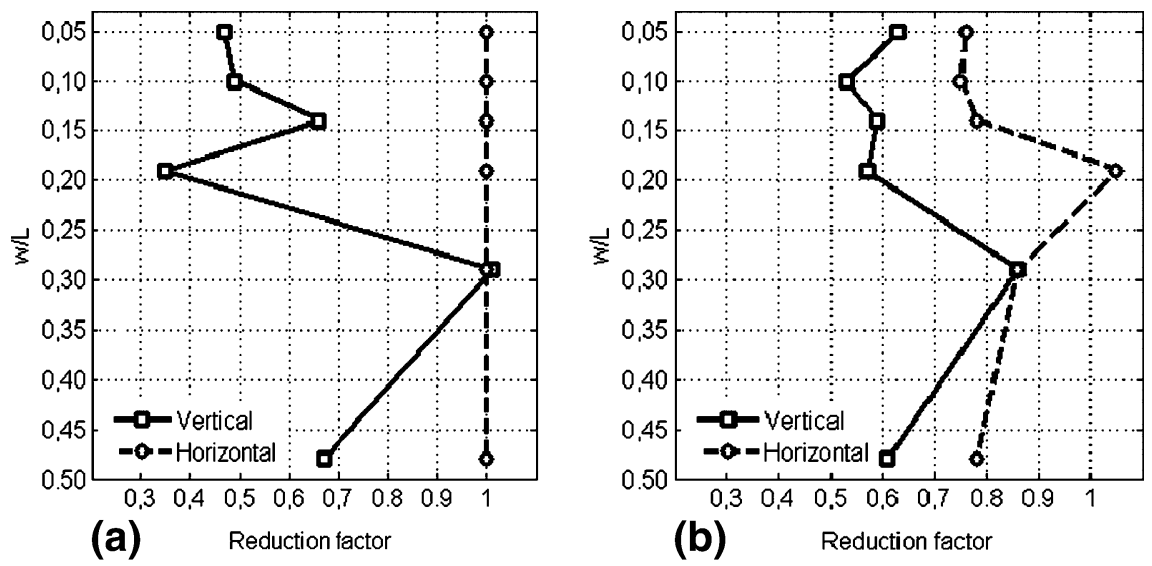

Figure 7. The variation of reduction factors (RF) with respect to $w / L$ ratios for obtained. (a) Displacement and (b) acceleration results. 
The screening performance of the isolation measure will be evaluated by using the vibration reduction factor $(\mathrm{RF})$ defined with following;

$$
R F_{\text {Displacement }}=\frac{P G D_{t}}{P G D_{c}}, R F_{\text {Acceleration }}=\frac{P G A_{t}}{P G A_{c}},
$$

where indexes $t$ is for various $w / L$ ratios and $c$ is stands in the case of no usage of WIB.

The variation of reduction factors (RF) with respect to $w / L$ ratios for obtained acceleration and displacement results are illustrated in figure 7. As could be seen, for the ratios of $w / L=0.19$
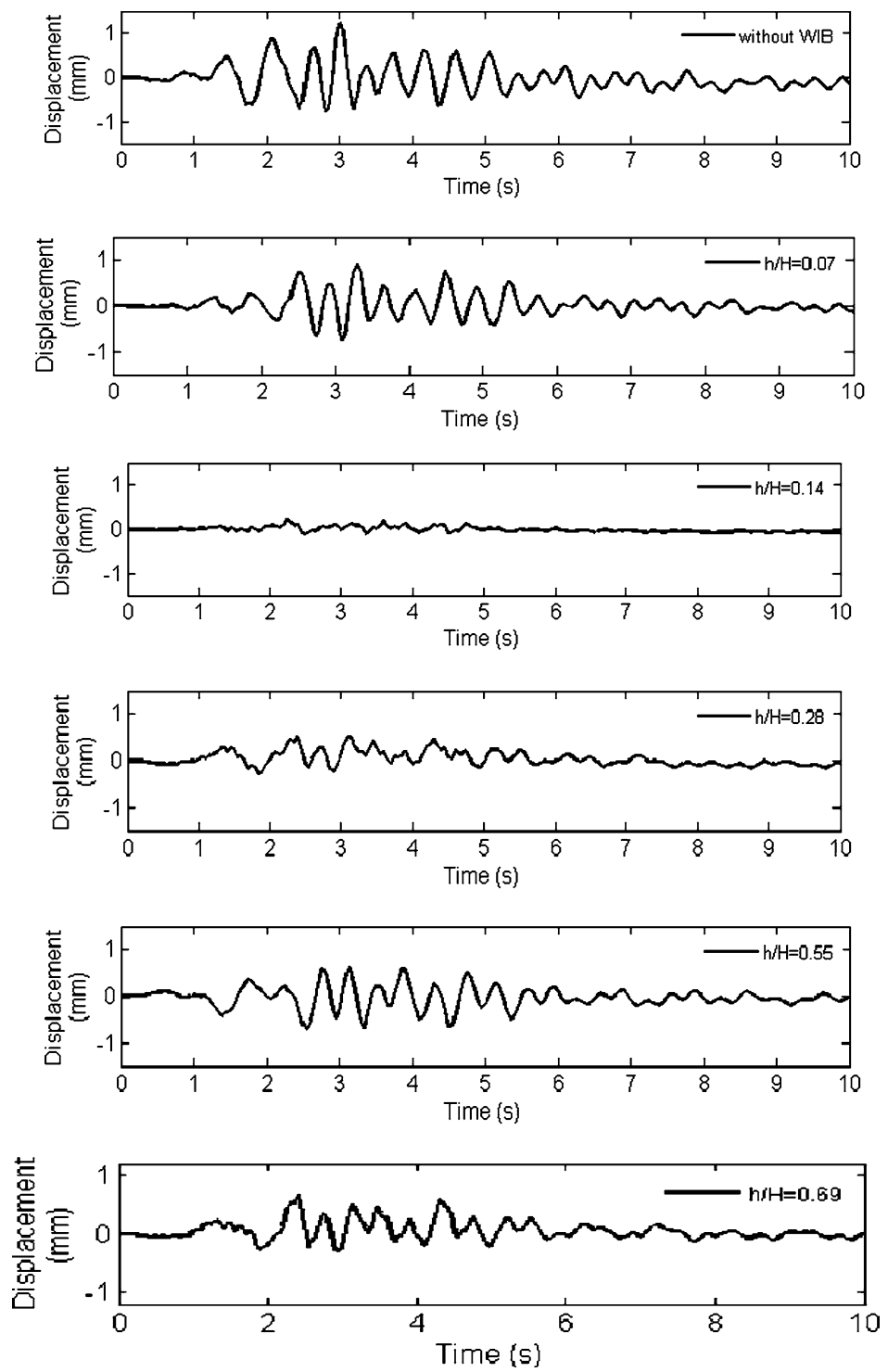

Figure 8. The vertical displacement time histories depending on $h / H$ ratios at measurement point A. 
and 0.10 gave the smallest RF for PGD and PGA, respectively. For the former, a $65 \%$ attenuation level is achieved in vertical displacement response where the latter is succeeded $47 \%$ reduction. Interestingly, only RF for $w / L=0.19$ is bigger than 1.00 which means wave barrier is amplified the horizontal acceleration response around 5\% for this thickness.

\subsection{Effect of the WIB depths, $h$}

In this part, the performance of the WIB in mitigating structural vibrations are examined with respect to the different depths of embankment varied from 20 to $200 \mathrm{~cm}$. Mixture of gravel and sand filling soil is used to model the filling above of embankment. A preferred controlling parameter which is obtained from the ratio of depth of embankment $(h)$ to height of trench $(H)$ is derived and proposed for the optimum thickness in reducing structural responses. The height of trench which is equal to $290 \mathrm{~cm}$ is assumed as summation of the radius of pipeline and filling upside down of the pipeline as $20 \mathrm{~cm}$ uniform fine sand and $100 \mathrm{~cm}$ mixture of gravel and sand is shown in figure 1 . Therefore $h / H$ ratios for aforementioned range of WIB's embankment depth are computed as $0.07,0.14,0.28,0.55$ and 0.69 , respectively.

The resulting time histories of the vertical displacement for various $h / H$ ratios considered with no WIB case are depicted in figure 8 . The obtained results indicate a remarkable reduction in the vertical amplitudes for any depth of embankment compared without WIB. The ratio with 0.14 provided the best screening effect considering PGD. From those time-domain responses, it should be noted that $40 \mathrm{~cm}$ is the most effective depth with $92 \%$ reduction. On the other hand, no affective reduction was observed for lateral structural vibrations. As shown in figure 9, the variations of horizontal displacements are plotted with shifted one centimeter. As in previous section, about $5 \mathrm{~cm}$ total displacements are seen in each case and WIB has no isolation effect in horizontal displacements.

The time dependent variations of vertical, horizontal acceleration and vertical displacement with respect to $h / H$ are plotted in figure 10. In terms of PGA, significant attenuating effect on

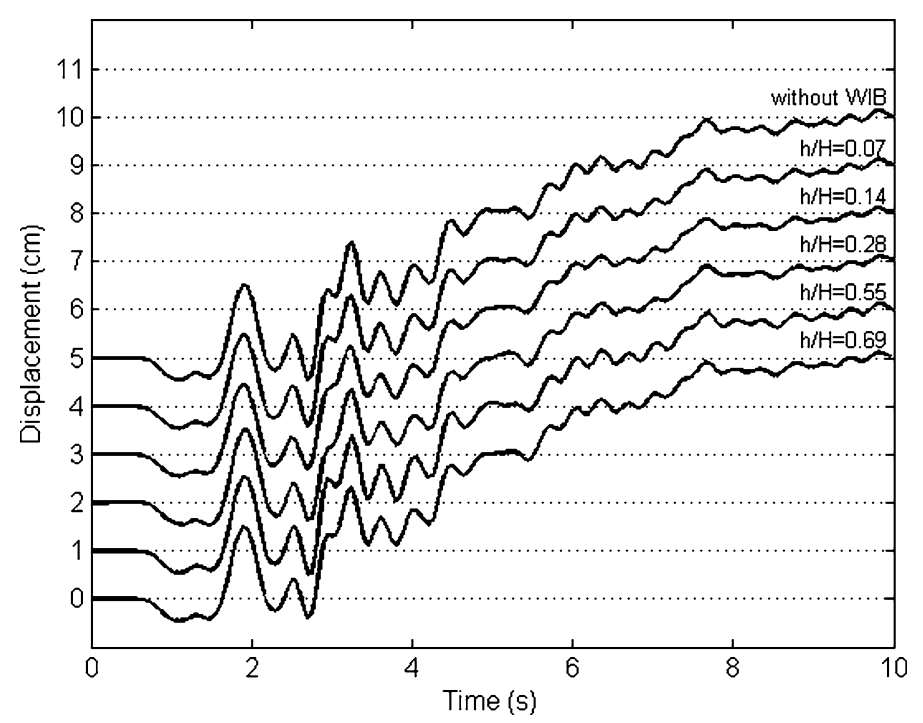

Figure 9. The horizontal displacement time histories depending on $h / H$ ratios at measurement point A (shifted record for $1 \mathrm{~cm}$ ). 


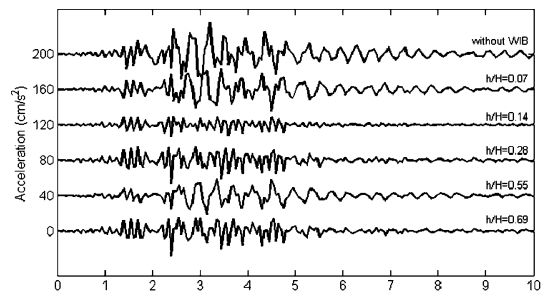

(a)

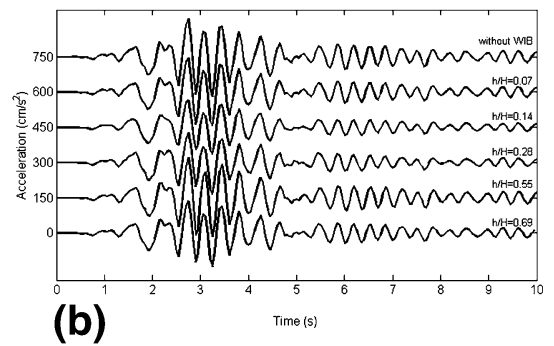

(b)

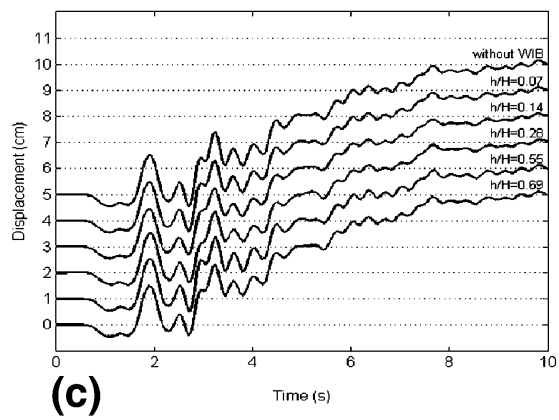

Figure 10. The resulting time histories of the (a) Vertical; (b) horizontal accelerations and (c) vertical displacement depending on various $\mathrm{h} / \mathrm{H}$ ratios (shifted records for $150 \mathrm{~cm} / \mathrm{s}^{2}, 40 \mathrm{~cm} / \mathrm{s}^{2}$ and $1 \mathrm{~cm}$ ).

structural response is acquired from the WIB for 0.14 of $h / H$ ratio. To denote explicitly the screening efficiency, the variation of reduction factors (RF) according to $h / H$ ratios for obtained displacement and acceleration results are displayed comparatively in figure 11.

Better isolation efficiency is performed similarly with ratio of $\mathrm{H} / \mathrm{h}=0.14$ for PGD and PGA. For the former, a promising reduction is accomplished with $92 \%$ and the latter is succeeded $64 \%$ mitigation in vertical acceleration response. Only for the ratio of $\mathrm{h} / \mathrm{H}=0.55$ has slight amplification effect on horizontal acceleration for this depth of embankment. A reduction level up to $32 \%$ of the horizontal acceleration could be observed for the depth of $\mathrm{H}=40 \mathrm{~cm}$. Analysing all cases, a general tendency is inferred that employment of wave impending barrier for buried pipelines is a very effective application to reduce the vibration. However, we should emphasize
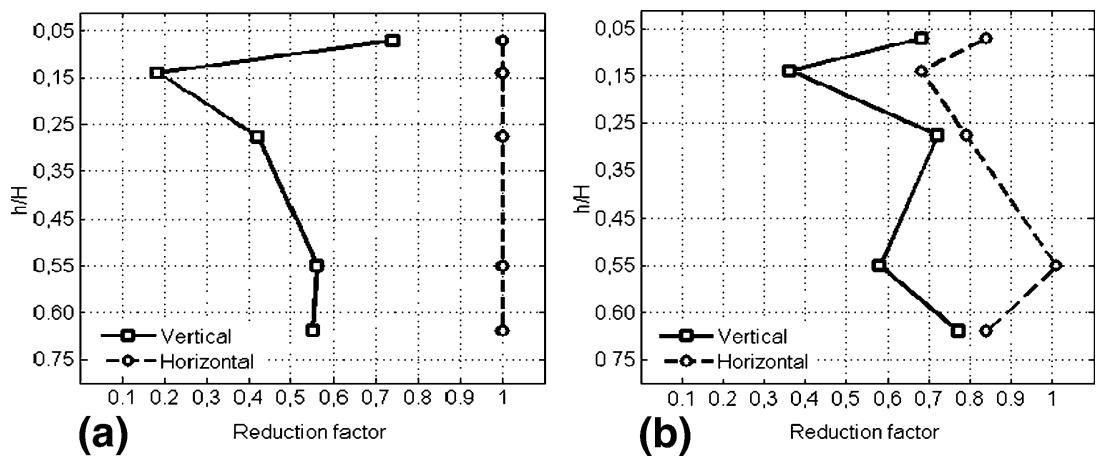

Figure 11. The variation of reduction factors (RF) with respect to $h / H$ ratios for obtained. (a) Displacement and (b) acceleration results. 
that reductions are obtained from near source record of 1990 Upland earthquake and might have dependency with respect to ground motion properties, thus a special care should be taken for amplification cases before practical application in this type of civil engineering problems.

\section{Conclusion}

The response of lifeline engineering under earthquake excitation which present a risk for population and environment is influenced by deformability of the buried pipelines such as used in natural gas transmission, especially in case of very soft ground conditions. In this study, computational simulation of the wave propagation problem with soil-structure interaction effects is directly performed by employing a two-dimensional (2D) plane-strain finite element model including nonlinear soil conditions with elasto-plastic Mohr-Coulomb constitutive law. Modelling capabilities of the implemented FE analysis with acceptable computational time and memory requirement are also taken into consideration for the numerical calculation of the infrastructural response of the buildings buried in soft ground to incident wave fields, induced by the earthquake excitation. This numerical model combined with simulation of the earthquake load in the time domain along with Newmark's integration completely takes into account the radiation damping condition along the fictitious interface of the unbounded media and directly predicts the shielding performance of the wave impeding barrier on the structural behaviour of the pipelines.

The effect of depth and width of WIB as an isolation measure, which is constructed underneath pipeline route, is investigated. Based on the numerical results, a concrete wave impeding barrier with optimum width, $65 \%$ and $47 \%$ reduction is achieved for PGD and PGA. Moreover, $92 \%$ and 64\% mitigation is accomplished for PGD and PGA with an optimum height of WIB. We have concluded that installing WIB has a significant attenuating effects on infrastructural response. Additionally, the geometrical parameters such as thickness and length of the wave barriers play an important role on the screening effectiveness.

\section{References}

Adam M and Chouw N 2001 Reduction of footing response to man-made excitations by using a wave impeding barrier. J. Appl. Mechanics 4: 423-431

Adam M and Estorff O Von 2005 Reduction of train-induced building vibrations by using open and filled trenches. Computers and Structures 83: 11-24

Ahmad S and Al-Hussaini T M 1991 Simplified design for vibration screening by open and infilled trenches. J. Geotechnical Eng. 117(1): 67-88

Beskos D E, Dasgupta G and Vardoulakis I G 1986 Vibration isolation using open or filled trenches part 1: 2-D homogeneous soil. Computational Mechanics 1(1): 43-63

Brinkgreve R B J, Al-Khoury R, Bakker K J, Bonnier P G, Brand P J W, Broere W, Burd H J, Soltys G, Vermeer P A and Haag D D 2002 Plaxis finite element code for soil and rock analyses, Published and distributed by A A Balkema Publisher, The Netherlands

Celebi E, Firat S and Cankaya I 2006 The effectiveness of wave barriers on the dynamic stiffness coefficients of foundations using boundary element method. Appl. Mathematics and Computation 180: 683-699

Celebi E, Fırat S, Beyhan G, Çankaya I, Vural I and Kírtel O 2009 Field experiments on wave propagations and vibration isolation by using wave barriers. Soil Dynamics and Earthquake Eng. 29: 824-833

Celebi E and Goktepe F 2012 Non-linear 2-D FE analysis for the assessment of isolation performance of wave impeding barrier in reduction of railway-induced surface waves. Construction and Building Materials 36(2012): 1-13 
Celebi E, Goktepe F and Kirtel O 2011 Vibration isolation measures for reductions of structural responses due passage of high-speed trains. Eurodyn 2011, Leuven, Belgium

Chouw N and Schmid G 1999 Numerical and experimental investigation on wave impediment in soil. Structural dynamics EURODYN'99, Fryba \& Naprstek, Balkema, Roterdam, pp. 977-982

Courant R, Friedrichs K and Lewy H 1967 On the Partial Difference Equations of Mathematical Physics. IBM J. 11: 215-234

Da Ha, Abdoun T H, O'Rourke M J, Symans M D, O'Rourke T D, Palmer M C and Stewart H E 2010 Earthquake Faulting Effects on Buried Pipelines-Case History and Centrifuge Study. J. Earthquake Eng. 14(5): 646-669

Daiyan N, Kenny S, Phillips R and Popescu R 2011a Numerical investigation of axial-vertical and lateralvertical pipeline/soil interaction in sand. Pan-Am CGS, Geotechnical Conference, Toronto, Ontario, Canada

Daiyan N, Kenny S, Phillips R and Popescu R 2011b Investigating pipeline-soil interaction under axiallateral relative movements in sand. Canadian Geotechnical J. 48(11): 1683-1695

Forchap E and Verbic B 1994 Wave propagation and reduction of foundation vibrations. Berg-Verlag GmbH, Bochum, pp. 165-178

Gamber N K 2004 Shallow foundation systems response to blast loading. A Thesis Presented to The Faculty of the Fritz J and Dolores H Russ College of Engineering and Technology, Ohio University, In Partial Fulfillment Of the Requirement for the Degree Master of Science, Athens

Goktepe F, Kirtel O and Celebi E 2010 Wave impeding block for mitigation of structural responses to train induced vibrations. 9th International Congress on Advances in Civil Engineering, Karadeniz Technical University, Trabzon, Turkey

Goktepe F, Kuyuk H S and Celebi E 2011a Efficiency of wave impeding barrier in pipeline construction under earthquake excitation using finite element analysis. International Conference on Earthquake Engineering and Seismology (ICEES: 2011), NUST, Islamabad, Pakistan

Goktepe F, Kuyuk H S and Celebi E 2011b Investigation on Wave Impending Barrier Thickness In Frequency Domain. Sixth International Conference of Seismology and Earthquake Engineering (SEE6), Tehran, Iran

Goktepe F, Kuyuk H S and Celebi E 2011c Efficiency of Wave Impeding Barrier for Wave Propagation In Pipeline under Earthquake Excitation. Seventh National Conference on Earthquake Engineering, Istanbul, Turkey (in Turkish)

Guo P 2005 Numerical Modeling of Pipe-Soil Interaction under Oblique Loading. J. Geotechnical and Geoenvironmental Eng. 131(2): 260-268

Hall W J and O'Rourke T D 1991 Seismic behavior and vulnerability of pipelines. Lifeline earthquake engineering Cassaro M A, (ed.) American Society of Civil Engineers, New York, pp. 761-773

Haupt W A 1981 Model tests on screening of surface waves. In: Proceedings of the 10th International Conference on Soil Mech. Found. Eng. Stockholm, Sweden, 3, pp. 215-222

Hughes T J R 1987 The finite element method. Prentice-Hall

Klein R, Antes H and Houedec D Le 1997 Efficient 3D modelling of vibration isolation by open trenches. Computers \& Structures 64(1-4): 809-817

Kouretzis G P, Bouckovalas G D and Gantes C J 2006 3-D shell analysis of cylindrical underground structures under seismic shear (S) wave action. Soil Dynamics and Earthquake Eng. 26(10): 909-921

Kouretzis G P, Sloan S W and Carter J P 2013 Effect of interface friction on tunnel liner internal forces due to seismic S- and P-wave propagation. Soil Dynamics and Earthquake Eng. 46: 41-51

Leung K L, Vardoulakis I G, Beskos D E and Tassoulas J L 1991 Vibration isolation by trenches in continuously non-homogenous soil by the BEM. Soil Dynamics and Earthquake Eng. 10(3): 172-179

Liu $\mathrm{H}$ and Song E 2005 Seismic response of large underground structures in liquefiable soils subjected to horizontal and vertical earthquake excitations. Computers and Geotechnics 32(4): 223-244

Pflanz G, Hashimoto K and Chouw N 2002 Reduction of structural vibrations induced by a moving load. J. Appl. Mechanics 5: 555-563

Smith I M and Griffith D V 1982 Programming the Finite Element Method. Second Edition, John Wiley \& Sons, Chisester, UK 
Takemiya H 1998a Lineside ground vibrations induced by high-speed train passage. Workshop on Effect of High-Speed Vibration on Structures and Equipment, Dept. Civil Eng., Nat. Cheng Kung Univ., Tainan, Taiwan, R.O.C., pp. 43-49

Takemiya H 1998b Paraseismic behavior of wave impeding block measured for ground vibration reduction. Workshop on Effect of High-Speed Vibration on Structures and Equipment, Dept. Civil Eng., Nat. Cheng Kung Univ., Tainan, Taiwan, R.O.C., pp. 51-56

Trifonov O V and Cherniy V P 2010 A semi-analytical approach to a nonlinear stress-strain analysis of buried steel pipelines crossing active faults. Soil Dynamics and Earthquake Eng. 30(10): 1298-1308

Woods R D 1968 Screening of surface waves in soils. J. Soil Mechanics and Foundations Division ASCE 94(4): 951-979

Zaneta G Adme 2006 Analysis NATM tunnel responses due to earthquake loading in various soils. Mining Technology Bulletin-Institute of Mining Science and Technology 2(3): 9-17

Zienkiewicz O and Taylor R 1991 The finite element method, 4th Edition. Vol 1, 2. McGraw-Hill, London 